\title{
Shear Thinning and Electro-Rheological Effect for Neat Liquid Crystalline Polysiloxane in the Vicinity of Isotropic-Liquid Crystalline Phase Transition
}

\author{
Katsufumi Tanaka, ${ }^{\dagger}$ Yasutaka Oiwa, Ryuichi Akiyama, and Atsushi Kubono \\ Department of Polymer Science and Engineering, \\ Kyoto Institute of Technology, Matsugasaki, Kyoto 606, Japan
}

(Received May 6, 1997)

\begin{abstract}
The phase transition behavior and the electro-rheological (ER) effect for a neat sample of the side chain type liquid crystalline polysiloxane were investigated in the vicinity of the isotropic-liquid crystalline phase transition temperature. The dependence of the dynamic viscoelastic properties upon temperature under no electric field changed at $308.1 \mathrm{~K}$ which was consistent with the isotropic-liquid crystalline phase transition temperature measured by the polarized optical microscopy. The shear thinning was observed under steady shear flow and no electric field at temperatures of $305 \mathrm{~K}$ as well as $313 \mathrm{~K}$. The ER effect was also observed at the same temperatures and the shear rates at which the shear thinning was observed. It was thought that the ER effect is closely related to the shear-induced alignment of anisotropic domains as well as mesogenic side groups under high shear fields before application of external electric fields.

KEY WORDS Electro-Rheology / Electro-Rheological Fluids / Liquid Crystalline Polymers / Phase Transition /
\end{abstract}

The electro-rheological (ER) fluids are known as the fluids which show the ER effect, that is, large enhancement in apparent viscosity and recovery to the original viscosity by application and removal of an external electric field, respectively. ${ }^{1-3}$ The ER fluids based on liquid crystalline polymers, the side chain type as well as the main chain type, ${ }^{4}$ have attracted intense interest since an outstanding breakthrough showing remarkable enhancement in shear stress induced by the electric fields was reported by Inoue and Maniwa ${ }^{5}$ using thermotropic liquid crystalline polymers of the side chain type. The ER fluids, which are composed of polysiloxane-based side chain liquid crystalline polymers diluted with polydimethylsiloxane, showed the stress enhancement in excess of $3000 \mathrm{~Pa}$ in absolute value and in excess of 10 times the steady shear stress under no electric field over a wide range of the shear rate, especially at high shear rates. ${ }^{6,7}$ It is explained that the ER effect of liquid crystals composed of small and rod-like molecules is caused mainly by the electro-hydrodynamic instability and the changes in contribution of the Miesowicz viscosities induced by external electric fields. ${ }^{2}$ Inoue and Maniwa ${ }^{6,7}$ pointed out that the weakness of the ER effect for the liquid crystals of small molecules can be caused by the weak interaction among anisotropic domains when the domains themselves are aligned in an electric field, thus making the domains unstable against mutual movement under shear. They used a side chain type liquid crystalline polysiloxane assuming that the polysiloxane main chains and flexible spacer chains in the side groups could effectively form a link between adjacent domains composed of mesogenic moiety. ${ }^{6,7}$ The use of the side chain type liquid crystalline polysiloxane is expected to result in the enhancement of the interaction among the domains under high shear flow. However, it is still unclear the mechanism of the ER effect for the fluids composed of the liquid crystalline polysiloxane diluted with poly-

\footnotetext{
† Author to whom correspondence should be addressed.
}

dimethylsiloxane (LCS/DMS).

One of the most important problems to be discussed is that the ER effect for LCS's/DMS is active at temperatures much higher than the critical temperature $\left(T_{\mathrm{c}}\right)$ of the isotropic phase-liquid crystalline phase transition for the neat sample of LCS observed by the differential scanning calorimetry (DSC) and the polarized optical microscopy (POM). Further, it is found that a diluted system of LCS is immiscible at a temperature much higher than $T_{\mathrm{c}}$. A new model of the ER effect is proposed for an immiscible blend on the basis of the microscopic observation $^{8}$ : The polymer (or oligomer) with high viscosity (LCS) is dispersed as droplets in the other polymer with low viscosity (DMS) in the absence of electric field, while the droplets are stretched and coalesced to form bridges between electrodes in the presence of electric field, resulting in the increase in the macroscopic viscosity. On the other hand, it is also pointed out that there can be miscible blends of LCS's/ DMS depending on the molecular structure of LCS's and the dilution of DMS. ${ }^{9}$ With all of the LCS's/DMS, the ER effect was observed at temperatures above their isotropic phase transition temperature, $T_{\mathrm{c}}$, as measured by DSC. ${ }^{9}$ It can be thought, as an explanation of the higher temperatures than $T_{\mathrm{c}}$ at which the ER effect of the LCS's/DMS is active, that the isotropic phase transition behavior of the neat LCS's changes in the presence of shear flow and external electric field. It is required for the discussion of the ER effect for the blended system in detail that the rheological and phase transition behaviors for the components of the blended system, especially for the neat LCS's, are clarified in the presence of shear flow and external electric field.

In the present paper, the phase transition behavior and the ER effect for a neat sample of the side chain type liquid crystalline polysiloxane were investigated in the vicinity of the isotropic-liquid crystalline phase transition temperature. 


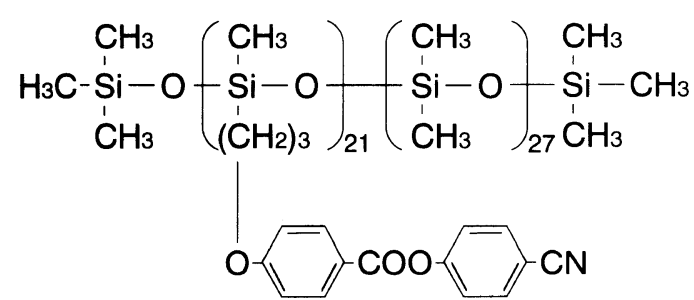

Figure 1. Chemical structure of the side chain type liquid crystalline polysiloxane (LCS) used in the present study.

\section{EXPERIMENTAL}

\section{Sample}

A neat sample of the side chain type LCS as shown in Figure 1 was kindly supplied from Asahi Chemical Industry Co., Ltd. The LCS was synthesized as reported in detail by the supplier..$^{5,10}$

The molecular weight distribution of the LCS dissolved in chloroform was characterized by gel permeation chromatography (GPC). The GPC was performed with a liquid chromatograph (Shimadzu, LC-10AD) equipped with a column oven (Shimadzu, CTO-10A), a UV spectrophotometric detector (Shimadzu, SPD-10A), and a data processing unit (Shimadzu, C-R4AX). A polystyrene gel column of Shodex GPC K-804L (Showa Denko) was used with chloroform as the eluent at 313 $\mathrm{K}$. The molecular weight was determined by calibrating with polystyrene standards. The polydispersity index $M_{w} / M_{n}$ was 2.1 . The weight average molecular weight was deduced to be $1.9 \times 10^{4}$ using the polystyrene standards.

All of the measurements in the present study were performed with the neat sample of the LCS. The neat LCS was opaque with a fluidity even at room temperature.

\section{Characterization of Phase Behavior}

The transition temperatures of the LCS were measured with a differential scanning calorimeter (Perkin-Elmer, DSC-7) under a dry nitrogen purge. The rate of heating and cooling was $2 \mathrm{~K} \mathrm{~min}^{-1}$. The texture of the LCS was observed with a polarized optical microscope (Olympus, $\mathrm{BH}-2$ ) with crossed polarizers equipped with a hot stage (Mettler, FP82) and a central control processor (Mettler, FP80). The magnification was $10 \times$ or $40 \times$, and the rate of heating and cooling was $0.1 \mathrm{~K} \mathrm{~min}^{-1}$. In the cooling process, time development of the texture was also observed at constant temperatures in the vicinity of the isotropic-liquid crystalline phase transition. The wide angle X-ray diffraction measurement was performed at room temperature with an X-ray diffractometer (Rigakudenki Ltd., RINT 2400) and an imaging plate (Rigakudenki Ltd., R-AXIS DS III) using a pinhole-collimated $\mathrm{Cu}-K_{\alpha}$ radiation.

\section{Rheological Measurements}

The temperature dependence of the dynamic viscoelastic properties such as storage modulus $G^{\prime}$, dynamic viscosity $\eta^{\prime}$, and loss tangent $\tan \delta$ in a cooling process under no electric field was measured by a rotational rheometer (Rheology Co., Ltd., MR-300V2E), a maximum torque of which was $2.94 \times 10^{-2} \mathrm{Nm}^{-1}$, with a fixture of cone and plate. The stress responses under constant shear rates and temperatures were also measured by the rheometer in the presence and absence of external electric field. A fixture of parallel plates was used as electrodes when an electric field was applied to the LCS. The LCS was deformed at a shear rate to be a steady flow. The sheared LCS was stimulated by stepwise electric fields with amplitudes up to $4.0 \mathrm{kV}$ $\mathrm{mm}^{-1}$. The stepwise electric fields were applied to the LCS in the direction perpendicular to the parallel plates using a piezo-drive amplifier driven by a signal generator. The detected signals of stress response were digitized by a 12-bits $A / D$ converter, and the data were stored in a personal computer.

\section{RESULTS AND DISCUSSION}

\section{Phase Behavior of LCS}

The transition temperature, $T_{\mathrm{c}}$, from the isotropic phase to the liquid crystalline phase in which fine-grain textures were observed by POM was $307.7 \mathrm{~K}$ in the cooling process. In the heating process, however, no critical phase transition to the isotropic phase and co-existence of the liquid crystalline phase and the isotropic phase were observed up to higher temperatures than $T_{\mathrm{c}}$. The DSC thermogram of the LCS for the second run heating as shown in Figure 2(a) exhibits a broad endothermic peak around $300 \mathrm{~K}$ after a base line shift around $290 \mathrm{~K}$. In the present paper, attention is focused on the results obtained in the cooling process. (Experimentally, temperature of the LCS was controlled with sufficient time and care for the rheological measurements.) Figure 2(b) shows the DSC thermogram of the LCS for the second run cooling. In Figure 2(b), two exothermic peaks are seen. The onset temperature of the exothermic peak of $304.6 \mathrm{~K}$ is about $307 \mathrm{~K}$ which agrees well with the isotropization temperature, $T_{\mathrm{c}}$, measured by POM although the cooling rate for the POM observation was much slower than that for the DSC measurement.

Below the isotropization temperature, the LCS exhibits a liquid crystalline mesophase. Probably, the liquid crystalline mesophase undergoes a very narrow smecticsmectic transition at $302.3 \mathrm{~K}$ corresponding to another exothermic peak in Figure 2(b), followed by the glass transition around $290 \mathrm{~K}$. The smectic mesophase below $302.3 \mathrm{~K}$ was assigned by the $\mathrm{X}$-ray diffraction as discussed later. However, only the POM hardly discriminated and assigned these two mesophases below the isotropization temperature because the characteristic textures of smectic or nematic phase ${ }^{11}$ were not found and only fine-grain ones were observed at the corresponding temperature range. The characterless textures in the liquid crystalline phase can be related to the relatively low substitution ratio of the mesogenic moiety. ${ }^{12}$ Together with the temperature dependence of the dynamic viscosity and the apparent viscosity around the isotropization temperature as discussed in the next section, we consider at the present stage that the transition from the isotropic phase to the liquid crystalline phase of the LCS is an isotropic-smectic phase transition, followed by a narrow smectic-smectic phase transition in the cooling process. However, it should be noted that the exothermic peak 


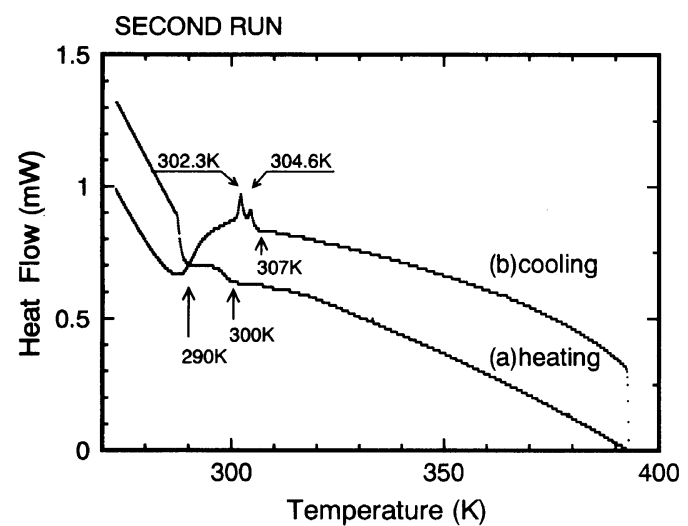

Figure 2. DSC thermograms of the LCS for the second run heating, (a), and the second run cooling, (b). The rate of heating and cooling was $2 \mathrm{~K} \mathrm{~min}^{-1}$

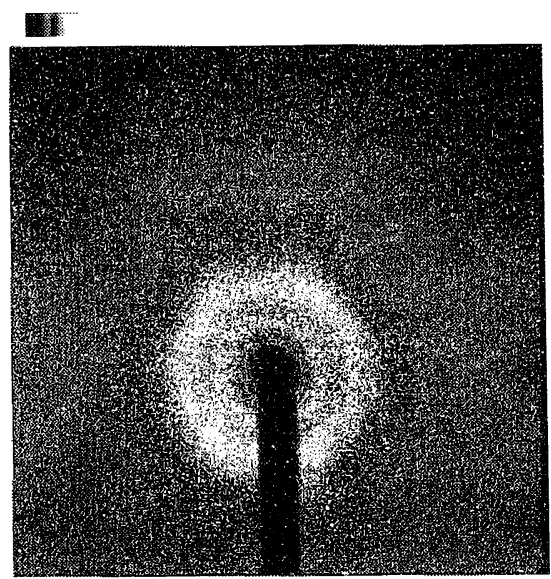

(a)

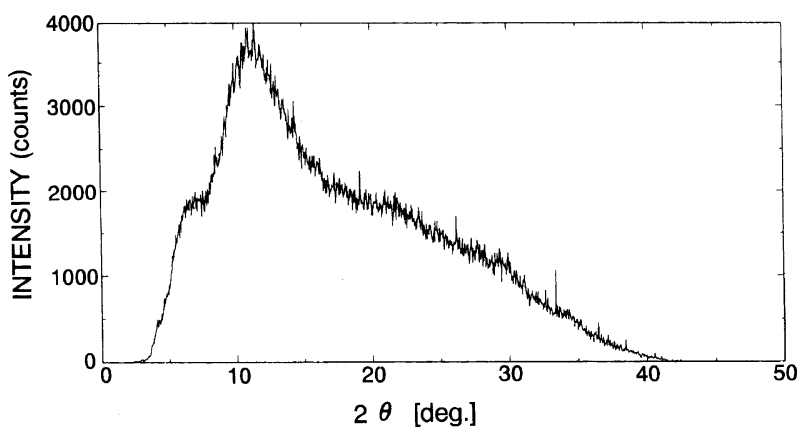

(b)

Figure 3. X-Ray diffraction pattern, (a), and its equatorial intensity profile, (b), of the LCS measured at room temperature (around $293-298 \mathrm{~K})$.

behavior of the LCS in the DSC thermogram depended remarkably on the cooling rate: At a cooling rate of $10 \mathrm{~K} \mathrm{~min}^{-1}$, a broad peak around $307 \mathrm{~K}$ corresponding to the peak at the higher temperature in Figure 2(b) was found with a broad shoulder below $304 \mathrm{~K}$ corresponding to the peak at the lower temperature. Therefore, we consider that the phase transition behavior as discussed in Figure 2(b) is not related to the supercooling of the LCS but it can be related to the distribution of the molecular weight of the LCS or local distribution of the substitution of the mesogenic moiety.

Figure 3 shows the X-ray diffraction pattern, (a), and its equatorial intensity profile, (b), measured at room

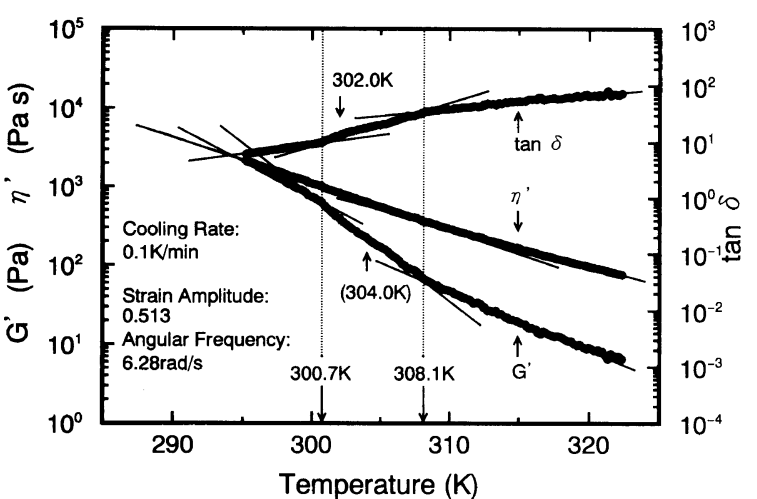

Figure 4. Temperature dependence of the dynamic viscoelastic properties of the LCS under no electric field. The cooling rate was $0.1 \mathrm{Kmin}^{-1}$.

temperature (around 293-298 K) at which the LCS was opaque with a fluidity. A weak smaller angle reflection at $2 \theta=6.95^{\circ}$ and a strong broad wider angle reflection at $2 \theta=11.5^{\circ}$ are found in the intensity profile. Further, a diffuse halo around $2 \theta=20^{\circ}$ is found. However, the diffuse halo with the corresponding $d$-spacing around $0.45 \mathrm{~nm}$ can be attributed to the reflection from a quartz glass capillary tube used as a sample holder although the $d$-spacing around $0.45 \mathrm{~nm}$ corresponds to the reflection from the intermolecular distance of the side chains along to the direction perpendicular to the molecular axes. ${ }^{11}$ The corresponding $d$-spacing at the smaller angle reflection is $1.27 \mathrm{~nm}$, which is characteristic of smectic phases. It is related to the layer spacing or the second order layer spacing. The total length of the side chain of the LCS is estimated assuming the fully extended conformation to be $1.765 \mathrm{~nm} .{ }^{13}$ The layer spacing of $1.27 \mathrm{~nm}$ is much shorter than the side chain length of the fully extended conformation. Therefore, an interdigitated bilayer packing model ${ }^{12}$ of the side chains, or a tilted packing model ${ }^{13}$ of the side chains in monolayer or bilayer is proposed in the present study. On the other hand, the corresponding $d$-spacing at the wider angle reflection is $0.77 \mathrm{~nm}$, which is much longer than a typical distance between the side chains around $0.45 \mathrm{~nm}$. The longer $d$-spacing might be related to the relatively low substitution ratio of the mesogenic moiety and short spacer length of three methylene units. However, the broad wider angle reflection can be attributed to the third order layer spacing ${ }^{12}$ assuming a bilayer packing model of the side chains although the three times the $d$-spacing of $0.77 \mathrm{~nm}$ was not necessarily in good agreement with the twice the $d$-spacing of the smaller angle reflection of $1.27 \mathrm{~nm}$. In addition, the similar broad reflection around $2 \theta=10^{\circ}$ was also observed for a liquid crystalline polysiloxane with an oligo(ethylene oxide) unit in the side chain. ${ }^{13}$ At any rate, the characteristic smaller angle reflection reveals the liquid crystalline phase to be a smectic phase at room temperature.

\section{Rheological Properties of LCS in the Presence and $A b$ - sence of Electric Fields}

Figure 4 shows the temperature dependence of the dynamic viscoelastic properties under no electric field. The stimulus of sinusoidal strain was thought to be 


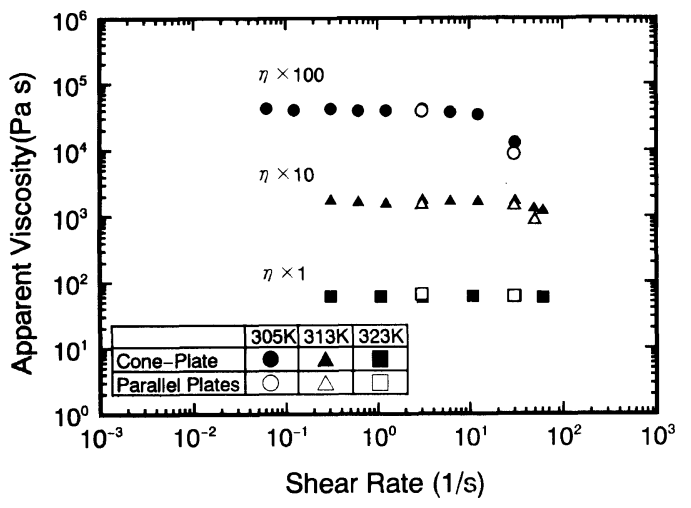

Figure 5. Shear rate dependence of the apparent viscosity of the LCS at constant temperatures under no electric field.

sufficiently small with low frequency compared with the steady shear flow at which the ER effect of the LCS's/DMS system is active. The linear response of stress was obtained over the cooling process at the present experiment. The cooling rate was $0.1 \mathrm{~K} \mathrm{~min}^{-1}$ which was assumed to be quasi-thermal equilibrium. In Figure 4, changes in the slopes of the dynamic properties at temperatures of $308.1 \mathrm{~K}, 302.0 \mathrm{~K}$, and $300.7 \mathrm{~K}$ are seen. Further, slight changes in the slopes around $304.0 \mathrm{~K}$ might be seen in Figure 4. (The changes in the slopes around $304.0 \mathrm{~K}$ were found obviously when the similar dynamic viscoelastic measurement as shown in Figure 4 was performed using a $9.81 \times 10^{-2} \mathrm{~N} \cdot \mathrm{m}$ torque meter.) The temperature of $308.1 \mathrm{~K}$ is in good agreement with the isotropic phase-liquid crystalline phase transition temperature of $T_{\mathrm{c}}$ measured by POM at the same cooling rate as the dynamic viscoelastic measurement. Further, it is found that the changes in the slopes around $308.1 \mathrm{~K}$ and $304.0 \mathrm{~K}$ correlate well with the onset and the termination, respectively, for the exothermic peak of the isotropization in the DSC thermogram as discussed in Figure 2(b). It is also found that the changes in the slopes around $302.0 \mathrm{~K}$ and $300.7 \mathrm{~K}$ correlate well with the onset and the termination, respectively, for the exothermic peak in the DSC thermogram. In Figure 4, the dynamic viscosity increases monotonically with a decrease in temperature although the dynamic viscosity is expected to decrease steeply below the isotropic phase-liquid crystalline phase transition temperature, especially for the case of the nematic phase transition. It is thought that the stimulus of sinusoidal strain was small enough not to align the mesogenic side chains along to the shear flow on a large scale, or that the interaction between the side chains was strong enough to form fine-grain domains, resulting in the opposite tendency of the dynamic viscosity. Similar tendency of the monotonic increase in the apparent viscosity as shown in Figure 4 was found around the isotropization temperature under a weak steady shear flow. Therefore, we consider at the present stage that the transition from the isotropic phase to the liquid crystalline phase of the LCS is an isotropic-smectic phase transition, followed by a narrow smectic-smectic phase transition in the cooling process. However, the phase transition behavior can be also related to the distribution of the molecular weight of the LCS or local distribution of the substitution of the

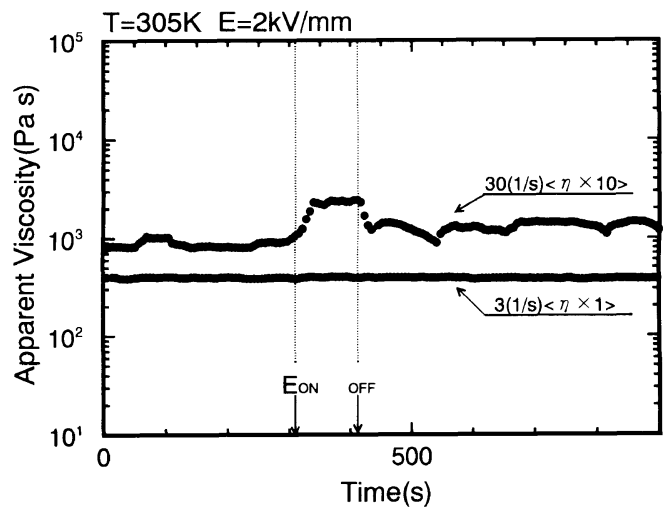

Figure 6. Changes in the apparent viscosity of the LCS at a temperature of $305 \mathrm{~K}$ in the presence and absence of electric field.

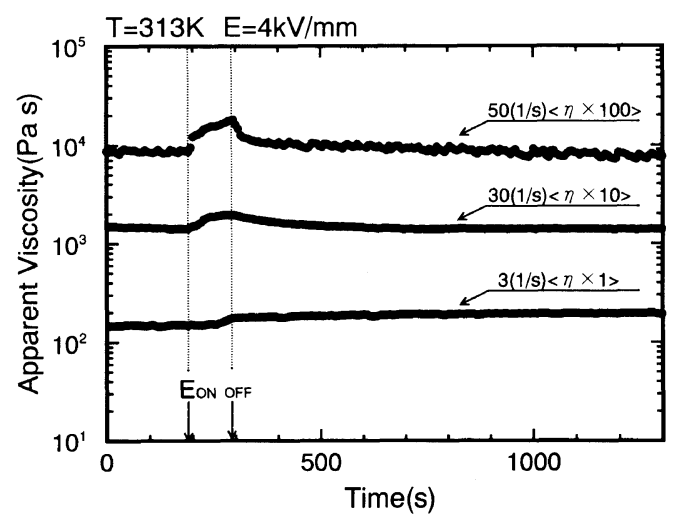

Figure 7. Changes in the apparent viscosity of the LCS at a temperature of $313 \mathrm{~K}$ in the presence and absence of electric field.

mesogenic moiety as mentioned in the previous section.

Figure 5 shows the shear rate dependence of the apparent viscosity for the LCS at constant temperatures under no electric field. It is found in Figure 5 that the apparent viscosity measured using a fixture of cone and plate agrees well with the apparent viscosity measured using that of parallel plates in the present experiment. The shear thinning is also found at temperatures of $305 \mathrm{~K}$ (below $T_{\mathrm{c}}$ ) and $313 \mathrm{~K}$ (above $T_{\mathrm{c}}$ ) at higher shear rates. On the other hand, no shear thinning is found at a temperature of $323 \mathrm{~K}$ even at higher shear rates. Figures 6 and 7 show changes in the apparent viscosity of the LCS at temperatures of $305 \mathrm{~K}$ and $313 \mathrm{~K}$, respectively, in the presence and absence of electric field. It is found in Figures 6 and 7 that the enhancement of the apparent viscosity is induced by external electric fields at the temperature of $305 \mathrm{~K}$ and even at the temperature of $313 \mathrm{~K}$ above $T_{\mathrm{c}}$ at the shear rates in the shear thinning range. Further, little or no ER effect was observed at the temperature of $323 \mathrm{~K}$ and at higher shear rates in the presence of electric fields up to $4 \mathrm{kV} \mathrm{mm}^{-1}$. Similar tendencies as shown in Figures. 5, 6, and 7 were obtained when the $9.81 \times 10^{-2} \mathrm{~N} \mathrm{~m}^{-1}$ torque meter was used for the rheological measurements. It is worthy to note that the ER effect observed above and below $T_{\mathrm{c}}$ is closely related to the shear thinning behavior of the LCS. It is thought that the ER effect in the liquid crystalline phase is closely related to the electrical orientation (perpendicular to the flow as shown in Figure 8) of anisotropic 


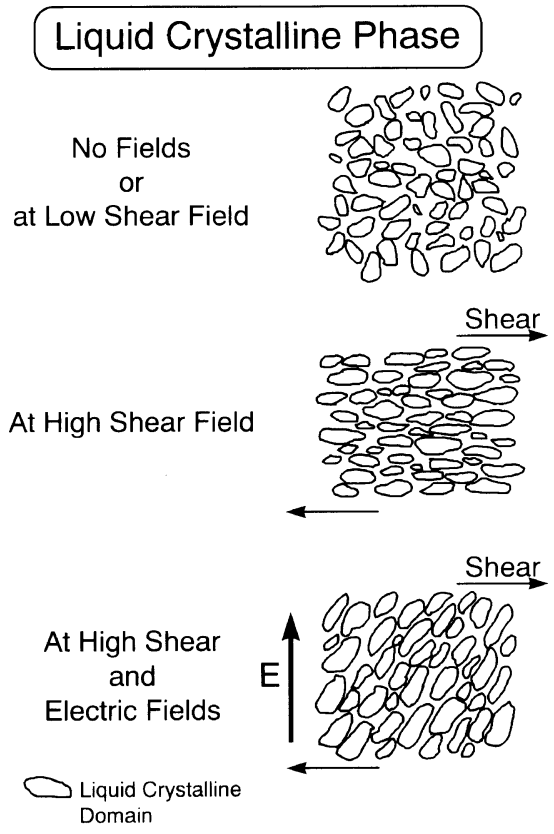

Figure 8. Schematic illustration of the mechanism of the ER effect for the LCS in the liquid crystalline phase.

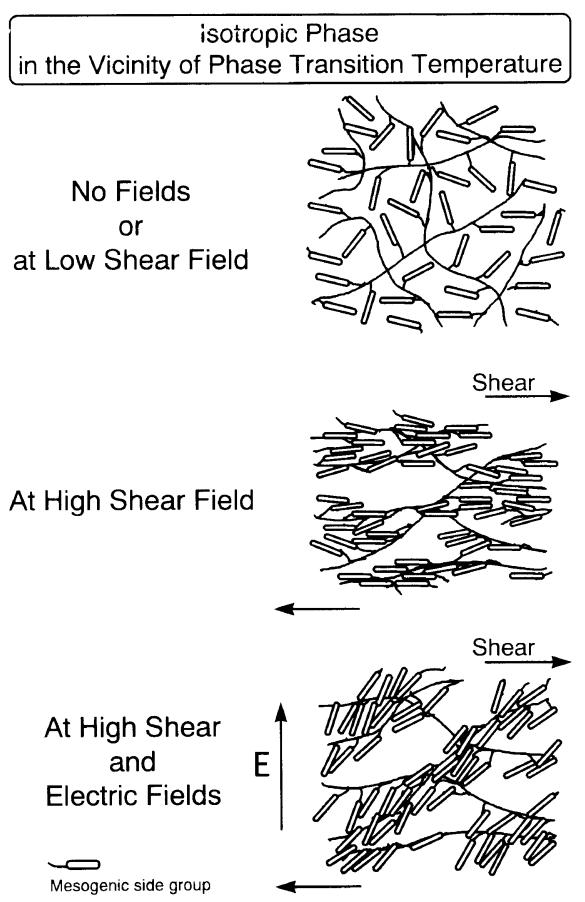

Figure 9. Schematic illustration of the mechanism of the ER effect for the LCS in the isotropic phase in the vicinity of the isotropic-liquid crystalline phase transition.

domains which has been aligned to the high shear flow before application of external electric fields. (We consider that the electro-hydrodynamic instability ${ }^{2,4}$ was negligible because of little or no ER effect as shown in Figure 6 at the lower shear rate of $3 \mathrm{~s}^{-1}$. If the electrohydrodynamic instability is not negligible, the LCS in the liquid crystalline phase should show the ER effect.) Also, anisotropic domains of the mesogenic side chains can be formed even at a temperature higher than $T_{\mathrm{c}}$ as shown in Figure 9, and that anisotropic domains are oriented to the flow direction under sufficiently high shear flow and no electric field. Consequently, it is thought that the ER effect observed above $T_{\mathrm{c}}$ is caused by the electrical orientation of the anisotropic domains which can be formed and aligned preferentially to the shear flow. However, the ER effect observed in the present study was two or three times the apparent viscosity under no electric field, which is somewhat smaller than the ER effect observed for an LCS/DMS diluted system.

From the results of the neat LCS compared with the diluted system of LCS's/DMS at temperatures much higher than $T_{\mathrm{c}}$, it is also considered that the mechanism of the ER effect in the fully isotropic phase for the diluted system of LCS's at temperatures much higher than $T_{\mathrm{c}}$ will be different from not only the mechanism in the liquid crystalline phase but also the mechanism at higher temperatures in the vicinity of the isotropic phase-liquid crystalline phase transition for the neat LCS: In the fully isotropic phase, the macroscopic structures such as the immiscibility or miscibility and their changes under external modulation of the shear flow as well as the electric fields will play an important role in the ER effect. Further investigations should be needed to clarify the mechanisms of the ER effect for the promising materials with scientific interests based on liquid crystalline polymers.

\section{CONCLUSIONS}

The phase transition behavior and the ER effect for a neat sample of the side chain type LCS were investigated in the vicinity of the isotropic-liquid crystalline phase transition temperature, $T_{\mathrm{c}}$. The dependence of the dynamic viscoelastic properties upon temperature under no electric field changed at temperatures which are closely related to the phase transitions of the neat LCS. The shear thinning was observed under steady shear flow and no electric field at temperatures above and below $T_{\mathrm{c}}$. The ER effect was also observed at the same temperatures and the shear rates at which the shear thinning was observed. It should be noteworthy that the ER effect was found even at a temperature above $T_{\mathrm{c}}$. It is thought that the ER effect is closely related to the shear-induced alignment of anisotropic domains as well as mesogenic side groups under high shear fields before application of external electric fields. However, it is also thought that the mechanism of the ER effect for the diluted system of LCS's/DMS at temperatures much higher than $T_{\mathrm{c}}$ will be different from that for the neat LCS.

Acknowledgments. The authors are grateful to Prof. K. Koyama (Yamagata University) for the DSC measurements, and to Dr. Y. Kawahara (Tokyo Metropolitan Textile Research Institute) for the X-ray diffraction. The authors are also grateful to Prof. K. Yonetake (Yamagata University) for fruitful discussion.

\section{REFERENCES}

1. H. Block and J. P. Kelly, J. Phys. D, 21, 1661 (1988), and references therein.

2. T. Honda, K. Kurosawa, and T. Sasada, Jpn. J. Appl. Phys., 18, 1015 (1979).

3. K. Tanaka, A. Fujii, and K. Koyama, Polym. J., 24, 995 (1992).

4. K. Tanaka, R. Akiyama, and K. Takada, Polym. J., 28, 419 


\section{K. TANAKA et al.}

(1996).

5. A. Inoue and S. Maniwa, Eur. Patent EP-0478034A1 (1992).

6. A. Inoue and S. Maniwa, J. Appl. Polym. Sci., 55, 113 (1995).

7. A. Inoue and S. Maniwa, J. Appl. Polym. Sci., 59, 797 (1996).

8. K. Tajiri, K. Ohta, T. Nagaya, H. Orihara, Y. Ishibashi, M. Doi, and A. Inoue, J. Rheol., 41, 335 (1997).

9. A. Inoue, S. Maniwa, and Y. Ide, Polym. Prepr., Jpn., 45, 3348 (1996).
10. A. Inoue, S. Maniwa, and Y. Ide, J. Appl. Polym. Sci., 64, 303 (1997).

11. S. Kibe, H. Kikuchi, and T. Kajiyama, Liquid Crystals, 21, 807 (1996).

12. K. Yonetake, M. Nakagomi, M. Ueda, and T. Masuko, Polym. J., 29, 240 (1997)

13. G. Chang-Chen and J. Kuo, Liquid Crystals, 20, 705 (1996). 